\title{
Sibs with mental retardation, supraorbital sclerosis, and metaphyseal dysplasia: frontometaphyseal dysplasia, craniometaphyseal dysplasia, or a new syndrome?
}

\author{
W Reardon, C M Hall, M J Dillon, M Baraitser
}

\begin{abstract}
A brother and sister are presented with unusual facies, bilateral mixed hearing loss, mental retardation, and widespread radiological abnormalities. The clinical and radiological evidence for and against the two most likely diagnoses of frontometaphyseal dysplasia and craniometaphyseal dysplasia is considered.
\end{abstract}

Significant radiological abnormalities of the skull and the metaphyseal ends of the long bones are features common to the conditions of frontometaphyseal dysplasia (McKusick No 30562) and craniometaphyseal dysplasia (McKusick No 12300 and 21840). ${ }^{1}$ Notwithstanding these similarities, it is usually possible to delineate these separate conditions based on clinical and radiological parameters.

Frontometaphyseal dysplasia is characterised by supraorbital prominence, hypertelorism, genu recurvatum, and small mandible. ${ }^{2}$ Deafness often occurs, probably as a result of cranial nerve compression. Mental retardation has been reported in one pair of half brothers, but intelligence is usually

Department of Clinical Genetics, The Hospital for Sick Children, Great Ormond Street, London WC1N 3JH.

W Reardon, M Baraitser

Department of Radiology, The Hospital for Sick Children, Great Ormond Street, London WCiN 3JH. C $M$ Hall

Department of General and Renal Medicine, The Hospital for Sick Children, Great Ormond Street, London WC1N 3JH.

M J Dillon

Correspondence to Dr Reardon, Institute of Medical Genetics, University of Wales College of Medicine, Heath Park, Cardiff CF4 4XN.

Received for publication 14 December 1991

Revised version accepted for publication 16 January 1991. normal. ${ }^{3}$ Although it has been suggested that inheritance may be autosomal dominant, the preponderance of males and the less severe phenotype in females suggest that $\mathrm{X}$ linked recessive inheritance is more likely. ${ }^{4}$

Craniometaphyseal dysplasia is characterised facially by hypertelorism and bony overgrowth at the root of the nose. It may be transmitted as an autosomal dominant or autosomal recessive condition. The striking radiological feature in both forms is the bony overgrowth at the base of the nose which leads to nasal obstruction and mouth breathing. Other features of facial nerve paresis, deafness, and external ophthalmoplegia are presumed to result from nerve compression. The basic pathology is thought to be a defect in osteoclastic activity so that normal bone resorption does not occur. ${ }^{5}$ Mental retardation has not been an observed feature of the condition.

This report presents details of a brother and sister with mental retardation and radiological abnormalities involving the skull and long bone metaphyses. The similarities and dissimilarities to frontometaphyseal dysplasia and craniometaphyseal dysplasia in these patients are discussed (table).

\section{Case reports}

Patients II 1 and II 2 are the offspring of clinically normal, non-consanguineous parents. Patient II 1 was born at 36 weeks of gestation and birth weight was $2250 \mathrm{~g}$. His motor milestones were delayed with crawling at 14 to 15 months and walking at 18 months. In consequence of a bilateral mixed hearing loss, he had his tonsils and adenoids removed at the age of 2 years. His hearing continued to deteriorate and, although he developed some speech, meaningful language usage was not achieved. Intellectual function was poor and formal IQ testing gave a score of $<50$.

The most recent clinical examination at the age of 17 years showed height on the 25 th centile, weight on the 10th centile, and head circumference on the 3rd centile. The striking physical feature was the 
Comparison of clinical and radiological features of frontometaphyseal dysplasia and craniometaphyseal dysplasia with those observed in the current report.

\begin{tabular}{|c|c|c|c|}
\hline & $\begin{array}{c}\text { Frontometaphyseal } \\
\text { dysplasia }\end{array}$ & $\begin{array}{c}\text { Craniometaphyseal } \\
\text { dysplasia }\end{array}$ & Current report \\
\hline \multicolumn{4}{|l|}{ Clinical features } \\
\hline Prominent supraorbital ridges & + & - & + \\
\hline Hypertelorism & + & + & + \\
\hline Thick nasal root and paranasal & - & ++ & - \\
\hline region & Hypolastic & Normal & Pronounced \\
\hline Mandible & + & + & + \\
\hline Hearing loss & + & - & - \\
\hline $\begin{array}{l}\text { Genu recurvatum } \\
\text { Mental retardation }\end{array}$ & Unusual & - & + \\
\hline Inheritance & ? X linked recessive & $A D$ and $A R$ forms & $?$ \\
\hline \multicolumn{4}{|l|}{ Radiological features } \\
\hline \multicolumn{4}{|l|}{ Skull } \\
\hline Supraorbital thickening & + & - & + \\
\hline Sclerosis of base and vault & + & + & ++ \\
\hline Obliteration of sinuses & + & + & + \\
\hline Thick bony nasal root & - & ++ & - \\
\hline Spine & Platyspondyly & Normal & Platyspondyly \\
\hline Ribs & Thin & Normal & Wide \\
\hline Metaphyses & Poorly modelled & Variable changes & Sclerotic and widened \\
\hline Pelvis & $\begin{array}{l}\text { Supra-acetabular } \\
\text { constriction }\end{array}$ & Normal & Narrow iliac wings \\
\hline
\end{tabular}

frontal bossing and hypertelorism. The forehead was broad and the supraorbital ridges prominent, especially laterally, giving the eyes a hooded appearance. The nasal bridge was flat, the tip broad, and the mandible prominent, giving the face a square profile (fig 1). Additionally the carrying angle of the arms was wide (fig 2). Radiological investigations in this patient showed marked sclerosis of the vault and base of the skull with pronounced thickening of the supraorbital region and obliteration of the frontal sinuses (fig 3). The metaphyses were sclerotic and widened (fig 4), with the knee, navicular, and carpal bones and the terminal phalanges of the hand being especially affected. Spinal radiographs showed a mild thoracic scoliosis, biconcave flattening of lumbar vertebral bodies, and vertebral endplate irregularity with disc space narrowing. The ribs were wide.
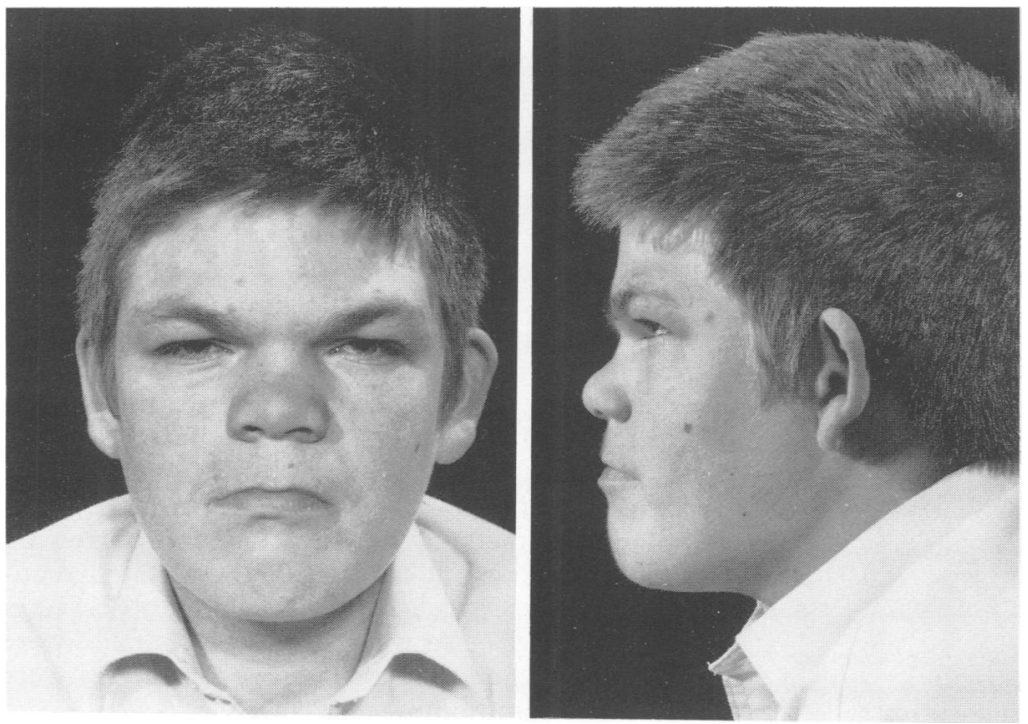

Figure 1 Patient II.1: note the prominent supraorbital ridges, frontal bossing, and hypertelorism. The mandible is prominent and well developed, giving the face a square profile. 


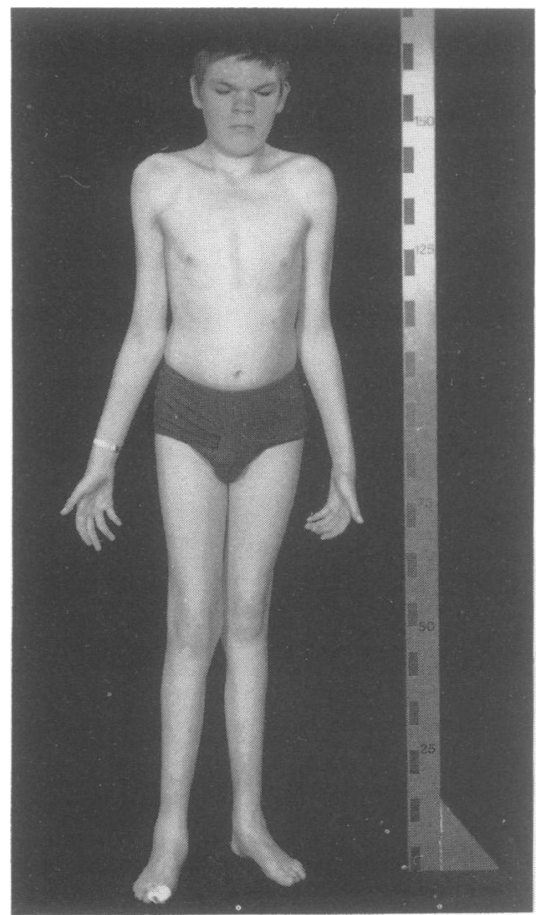

Figure 2 Patient II.1: note the increased carrying angle of the arms.

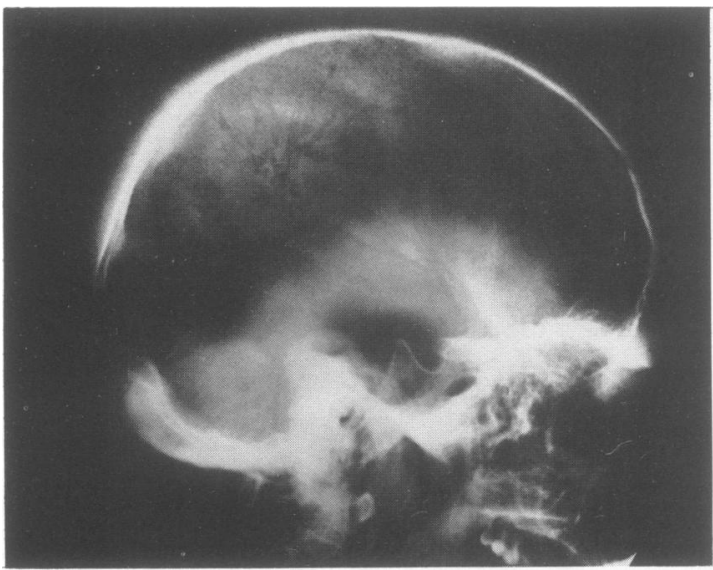

Figure 3 Patient II·1: lateral skull radiograph showing basal sclerosis and vault thickening, especially of the frontal and occipital bones. The frontal sinuses are absent.

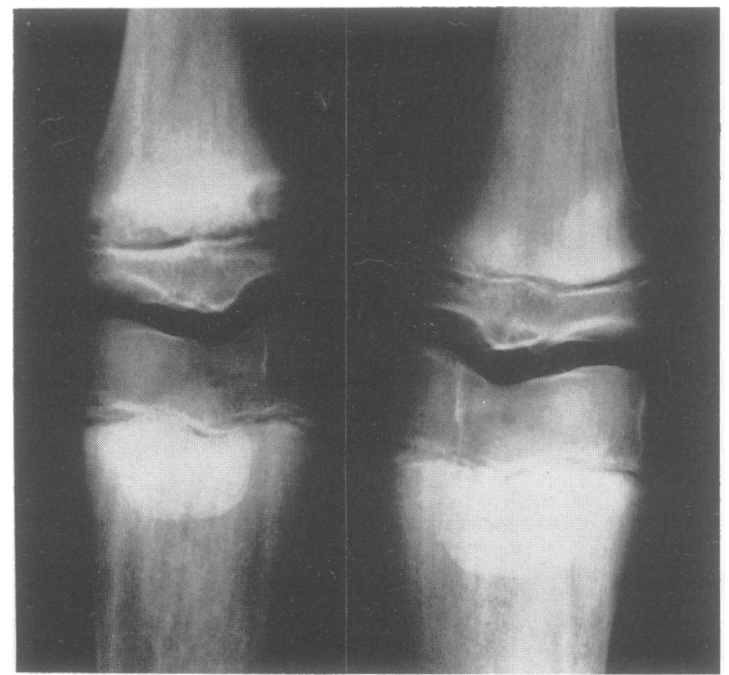

Figure 4 Patient II.1: anteroposterior radiograph of the knees. The metaphyses are wide, undermodelled, and sclerotic. The left upper tibial metaphysis is irregular, probably related to repeated stress.

Full biochemical profile, including urinary organic acids, was normal, as were chromosomal studies.

Patient II 2 is the younger sister of II 1 . She was born at 40 weeks' gestation after a normal pregnancy, birth weight $2500 \mathrm{~g}$. Early development appeared normal but a progressive hearing loss was confirmed at 4 years. However, this patient learned to lip read and to speak in sentences. She attended a special school and formal IQ evaluation gave a score of 70. Unlike her brother she did not need to use a hearing aid and she developed a much greater sense of social independence than he did.

Clinical examination aged 15 years showed height on the 75th centile, weight on the 50th centile, and head circumference marginally below the 50 th centile. Like her brother, she had hypertelorism, with pronounced supraorbital ridges, broad forehead and nasal tip, and a prominent mandible. The frontal hairline was normal (fig 5). The carrying angle of the arms was increased. Neurological examination was normal apart from deafness. Although milder than in her brother, the radiological features were undoubtedly of the same condition, with cranial sclerosis, supraorbital thickening, frontal sinus obliteration, metaphyseal sclerosis, and narrowing of the iliac wings (fig 6). Vertebral endplate irregularity with disc space narrowing was also observed in this patient (fig 7). As with her brother, her ribs were broad (fig 8) and all biochemical parameters were normal. Chromosomes were $46, \mathrm{XX}$. 

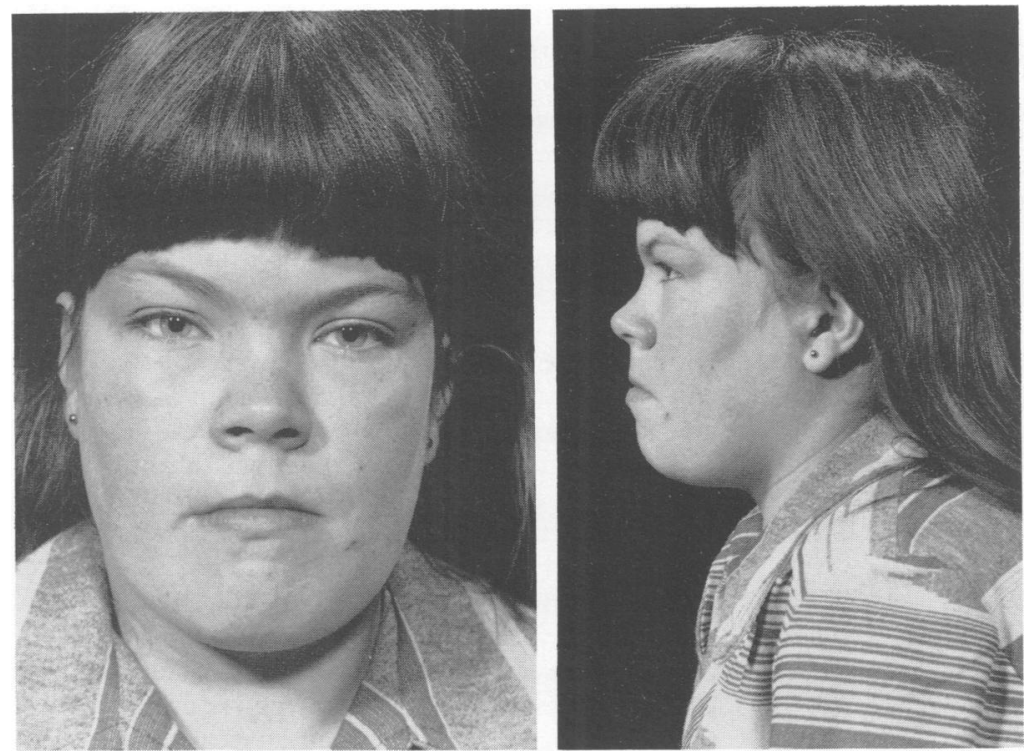

Figure 5 The facial features in patient $I I \cdot 2$.

\section{Discussion}

This brother and sister present an interesting diagnostic problem. The prominent supraorbital ridges, hypertelorism, and deafness all support a clinical diagnosis of frontometaphyseal dysplasia. Furthermore, the milder phenotype in the sister and the mild scoliosis in the brother, also reported in three previous cases, ${ }^{6-8}$ add weight to this diagnosis. However, neither patient has the genu recurvatum

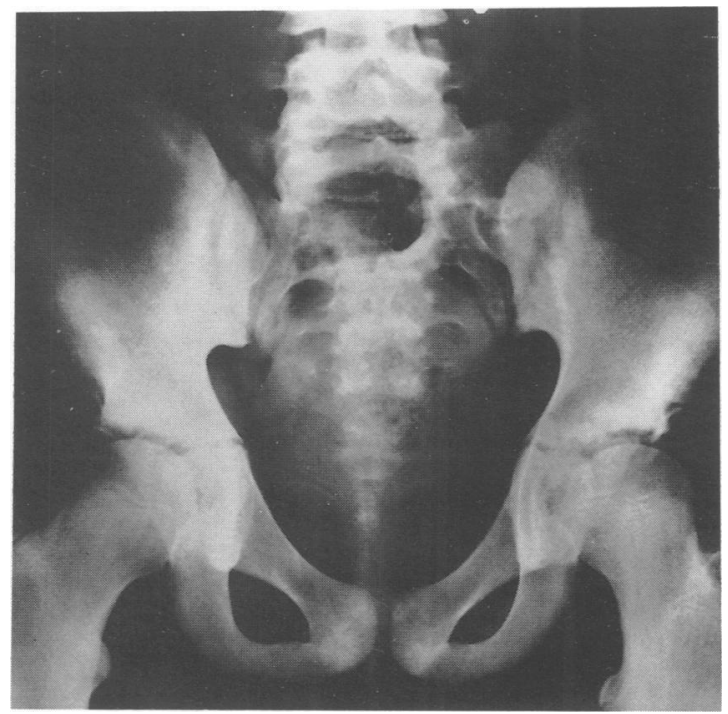

Figure 6 Patient II·2: anteroposterior view of pelvis. The iliac wings are narrow. Some coxa valga is present.

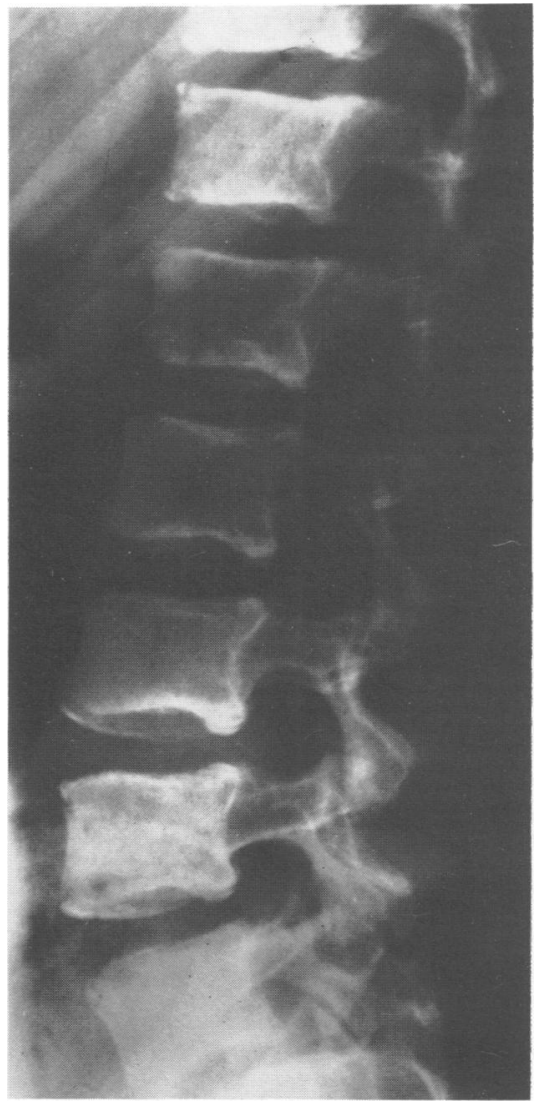

Figure 7 Patient II·2: lateral view of spine. There is mild vertebral endplate irregularity. 


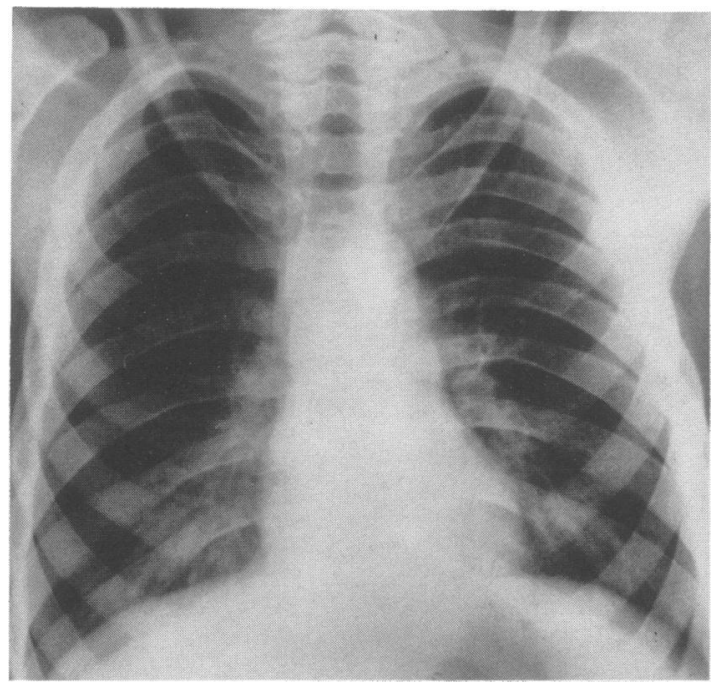

Figure 8 Patient $I I \cdot 2$ : anteroposterior radiograph of the chest. The ribs are uniformly widened.

or mandibular hypoplasia that also characterise this condition. Indeed, the mandible in these patients is prominent (figs 1, 3, and 5).

In general, cases reported with frontometaphyseal dysplasia have normal intelligence, although mental retardation has been described once. ${ }^{3}$ This report documented two half brothers, sharing a common mother, who had kyphoscoliosis, prominent supraorbital ridges, and were severely mentally retarded (IQ <20). Unlike the subjects of this report, these patients were very microcephalic and had small jaws. Their facial features were dominated by an inch thick osseous ridge supraorbitally, described as giving the face a 'visor'-like appearance. In contrast, the patients we describe have pronounced mandibles, a much milder degree of supraorbital ridge thickening, and neither of our patients satisfies the definition of microcephaly. Although patient II 1 does show microcephaly relative to other body parameters, it is not comparable to the microcephaly observed in the mentally retarded half brothers. ${ }^{3}$

In terms of radiology, the sclerosis of the skull vault and base, supraorbital thickening, sinus obliteration, vertebral endplate irregularities, and failure of metaphyseal modelling in the long bones which we have observed in these patients support a diagnosis of frontometaphyseal dysplasia. ${ }^{9}$ The appearances in our patients are not classical of frontometaphyseal dysplasia, however, in that the ribs are wide and the iliac wings narrow without the flaring and supra-acetabular constrictions usually associated with the condition. ${ }^{679}$

The alternative possible diagnosis is that of craniometaphyseal dysplasia. Mental retardation is not a feature of this condition and the absence of nasal root bony thickening in our patients is a further factor against this diagnosis. Although the cranial and metaphyseal radiological abnormalities in our patients are compatible with this diagnosis, the abnormalities of the spine and pelvis are not, since these are normal in craniometaphyseal dysplasia. ${ }^{9}$

For these reasons our patients do not fit into either of the two most likely diagnostic categories. We must conclude that either these patients represent a new syndrome or else that they represent a broadening of the phenotype of either frontometaphyseal dysplasia or craniometaphyseal dysplasia. Further case studies of these two conditions may help to resolve the issue.

1 McKusick VA. Mendelian inheritance in man. 8th ed. Baltimore: Johns Hopkins University Press, 1988.

2 Beighton $\mathrm{P}$, Hamersma H. Frontometaphyseal dysplasia: autosomal dominant or X linked? ₹ Med Genet 1980;17:53-6.

3 Jervis GA, Jenkins EC. Case report 31. Syndrome Identification 1975;3:18-20.

4 Gorlin RJ, Winter RB. Frontometaphyseal dysplasia - evidence for X-linked inheritance. Am $\mathcal{f}$ Med Genet 1980;5:81-4.

5 Millard DR, Maisels DO, Batstone JHF, Yates BW. Craniofacial surgery in craniometaphyseal dysplasia. Am $\mathcal{f}$ Surg 1967; 113:615-21.

6 Gorlin RJ, Cohen MM Jr. Frontometaphyseal dysplasia. $\operatorname{Am} \mathcal{F}$ Dis Child 1969;118:487-94.

7 Danks DM, Mayne V, Hall RK, McKinnon MC. Frontometaphyseal dysplasia. Am 7 Dis Child 1972;123:254-8.

8 Weiss L, Reynolds WA, Szymanowski RT. Frontometaphyseal dysplasia. Am $\mathcal{F}$ Dis Child 1976;130:259-61.

9 Wynne-Davies R, Hall CM, Apley AG. Atlas of skeletal dysplasias. 1st ed. Edinburgh: Churchill Livingstone, 1985. 